\title{
Statistical Analysis for Food Quality in the Presence of Vague Information
}

\author{
Ali Hussein Al-Marshadi and Muhammad Aslam \\ Department of Statistics, Faculty of Science, King Abdulaziz University, Jeddah 21551, Saudi Arabia \\ Correspondence should be addressed to Muhammad Aslam; aslam_ravian@hotmail.com
}

Received 20 June 2021; Accepted 6 September 2021; Published 17 September 2021

Academic Editor: Chunpeng Wan

Copyright (C) 2021 Ali Hussein Al-Marshadi and Muhammad Aslam. This is an open access article distributed under the Creative Commons Attribution License, which permits unrestricted use, distribution, and reproduction in any medium, provided the original work is properly cited.

\begin{abstract}
The present study introduces the neutrosophic statistical test to see investigate the difference between variances of two populations when correlation exits in pair observations. The procedure and statistic of the proposed test under neutrosophic statistics are introduced in the paper. The application of the proposed test is given using the food industry data. The efficiency of the proposed test is compared with that of the existing test in terms of the measure of indeterminacy, flexibility, and information. From the real application and comparative studies, it is concluded that the proposed test is quite reasonable to apply in uncertainty.
\end{abstract}

\section{Introduction}

The F-test is applied for testing the equality of two population variances under the assumption that the data are obtained from the normal distribution. Usually, the F-tests are applied under the assumption that the data are independent and obtained from the normal distribution [1]; we also discussed the application of the $F$-test when the pair data are correlated. This F-test is applied to investigate the significant difference between the equality of two population variances with correlated pair data [2-4]. More applications about the statistical tests can be seen in [4-8] where the applications of statistical tests in various practical fields are provided.

Classical statistics-based tests cannot be applied to the observations in the data which are fuzzy, in intervals, and uncertain. The fuzzy-based tests are the alternative of classical statistical tests in these situations. As mentioned in [9], "statistical data are frequently not precise numbers but more or less nonprecise, also called fuzzy. Measurements of continuous variables are always fuzzy to a certain degree." The authors of [10-22] worked on various types of the statistical tests using fuzzy logic.

The statistical tests based on fuzzy logic do not give information about the measure of indeterminacy. To overcome this shortcoming [23], we introduced neutrosophic logic as an extension of fuzzy logic. The efficiency of the neutrosophic logic over fuzzy analysis and intervalbased analysis was discussed in [24]. Recently, several applications of the neutrosophic logic were discussed in [25-30] and neutrosophic statistics were introduced as an extension of classical statistics. For more details on the neutrosophic statistics, see https://archive.org/details/ neutrosophic-statistics?tab=about, https://archive.org/ details/neutrosophic-statistics?tab=collection and http://fs. unm.edu/NS/NeutrosophicStatistics.htm. The efficiency of neutrosophic statistics over classical statistics was proven in [31-37].

The existing F-test for testing the equality of variances for correlated data under classical statistics cannot be applied in the presence of imprecise data. By exploring the literature and to the best of our knowledge, we did not find any work on the F-test for testing the equality of variances for correlated data under neutrosophic statistics. In this paper, the work when observations are correlated under neutrosophic statistics will be presented. The operational procedure of the proposed test will be given for testing the hypothesis of equality of two variances. The application of the proposed test will be given in the data taken from the food industry. It is expected that the proposed test will be efficient than the 
existing test in terms of information, flexibility, and adequacy.

\section{The Proposed F-Test for Variances with Correlated Data}

As mentioned before, the existing $F$-test for two population variances when pair data are correlated can be applied only when all observations are determined, certain, and exact. In this section, the $F$-test for two population variances when pair data are correlated will be introduced when the data is an interval, indeterminate, and neutrosophic. The main objective of the proposed test is to investigate the difference between two pupation variances when the data are paired and have a correlation. In addition, it is assumed that the data follow the neutrosophic normal distribution. Let $\sigma_{1 N}^{2}$ and $\sigma_{2 N}^{2}$ be the neutrosophic variances of the first and the second population, respectively. The proposed test will be applied for testing the null hypothesis. The proposed test will be applied for testing the null hypothesis $H_{0}: \sigma_{1 N}^{2}=\sigma_{2 N}^{2}$ vs. the alternative hypothesis $H_{1}: \sigma_{1 N}^{2} \neq \sigma_{2 N}^{2}$. Suppose that $\left\{X_{1 N}=X_{1 L}+X_{1 U} I_{1 N} ; \quad I_{1 X N} \in\left[I_{1 X L}, \quad I_{1 X U}\right], Y_{1 N}=Y_{1 L}+\right.$ $\left.Y_{1 U} I_{1 N} ; I_{1 Y N} \in\left[I_{1 Y L}, I_{1 Y U}\right]\right\}, \quad \ldots, \quad\left\{X_{n N}=X_{n L}+\right.$ $X_{n U} I_{n N} ; I_{n X N} \in\left[I_{n X L}, I_{n X U}\right], \quad Y_{n N}=Y_{n L}+Y_{n U} I_{n Y N} ; I_{n Y N}$ $\left.\in\left[I_{n Y L}, I_{n Y U}\right]\right\}$ is a pair of neutrosophic observations of neutrosophic sample size $n_{N} \in\left[n_{L}, n_{U}\right]$. Note that $X_{1 L}$, $\ldots, X_{n L}$ and $Y_{1 L}, \ldots, Y_{n L}$ are the determined part of pair observations and $X_{1 U} I_{1 N}, \ldots, X_{n U} I_{n X N}$ and $Y_{1 U} I_{1 N}, \ldots, Y_{n U} I_{n Y N}$ are the indeterminate part of the same pair observations. Note also that $I_{n X N} \in\left[I_{n X L}, I_{n X U}\right]$ and $I_{n Y N} \in\left[I_{n Y L}, I_{n Y U}\right]$ are measures of indeterminacy associated with the neutrosophic pair observations. Based on the information and by following the work in $[35,36]$, the neutrosophic means are defined as

$$
\begin{aligned}
& \bar{X}_{i N}=\bar{X}_{L}+\bar{X}_{U} I_{X N} ; I_{X N} \in\left[I_{X L}, I_{X U}\right] ; \quad i=1,2,3, \ldots, n_{N}, \\
& \bar{Y}_{i N}=\bar{Y}_{L}+\bar{Y}_{U} I_{Y N} ; I_{Y N} \in\left[I_{Y L}, I_{Y U}\right] ; \quad i=1,2,3, \ldots, n_{N} .
\end{aligned}
$$

The neutrosophic variances are defined as

$$
\begin{array}{cc}
S_{X N}^{2}=\frac{\sum_{i=1}^{n_{N}}\left(X_{i N}-\bar{X}_{i N}\right)^{2}}{n_{N}} ; & n_{N} \in\left[n_{L}, n_{U}\right], \bar{X}_{i N} \in\left[\bar{X}_{i L}, \bar{X}_{i U}\right], \\
S_{Y N}^{2}=\frac{\sum_{i=1}^{n_{N}}\left(Y_{i N}-\bar{Y}_{i N}\right)^{2}}{n_{N}} ; & n_{N} \in\left[n_{L}, n_{U}\right], \bar{Y}_{i N} \in\left[\bar{Y}_{i L}, \bar{Y}_{i U}\right],
\end{array}
$$

where

$$
\begin{aligned}
& \sum_{i=1}^{n_{N}}\left(X_{i}-\bar{X}_{i N}\right)^{2}=\sum_{i=1}^{n_{N}}\left[\begin{array}{c}
\min \left(\begin{array}{c}
\left(X_{i L}+X_{U i} I_{L}\right)\left(\bar{X}_{L}+\bar{X}_{U} I_{L}\right),\left(X_{i L}+X_{i U} I_{L}\right)\left(\bar{X}_{L}+\bar{X}_{U} I_{U}\right) \\
\left(X_{i L}+X_{i U} I_{U}\right)\left(\bar{X}_{L}+\bar{X}_{U} I_{L}\right),\left(X_{i L}+X_{i U} I_{U}\right)\left(\bar{X}_{L}+\bar{X}_{U} I_{U}\right)
\end{array}\right) \\
\max \left(\begin{array}{c}
\left(X_{i L}+X_{i U} I_{L}\right)\left(\bar{X}_{L}+\bar{X}_{U} I_{L}\right),\left(X_{i L}+X_{U} I_{L}\right)\left(\bar{X}_{L}+\bar{X}_{U} I_{U}\right) \\
\left(X_{i L}+X_{i U} I_{U}\right)\left(\bar{X}_{L}+\bar{X}_{U} I_{L}\right),\left(X_{i L}+X_{i U} I_{U}\right)\left(\bar{X}_{L}+\bar{X}_{U} I_{U}\right)
\end{array}\right)
\end{array}\right], \quad I_{X N} \in\left[I_{X L}, I_{X U}\right], \\
& \sum_{i=1}^{n_{N}}\left(Y_{i}-\bar{Y}_{i N}\right)^{2}=\sum_{i=1}^{n_{N}}\left[\begin{array}{c}
\min \left(\begin{array}{c}
\left(Y_{i L}+Y_{U i} I_{L}\right)\left(\bar{Y}_{L}+\bar{Y}_{U} I_{L}\right),\left(Y_{i L}+Y_{i U} I_{L}\right)\left(\bar{Y}_{L}+\bar{Y}_{U} I_{U}\right) \\
\left(Y_{i L}+Y_{i U} I_{U}\right)\left(\bar{Y}_{L}+\bar{Y}_{U} I_{L}\right),\left(Y_{i L}+Y_{i U} I_{U}\right)\left(\bar{Y}_{L}+\bar{Y}_{U} I_{U}\right)
\end{array}\right) \\
\max \left(\begin{array}{c}
\left(Y_{i L}+Y_{i U} I_{L}\right)\left(\bar{Y}_{L}+\bar{Y}_{U} I_{L}\right),\left(Y_{i L}+Y_{U} I_{L}\right)\left(\bar{Y}_{L}+\bar{Y}_{U} I_{U}\right) \\
\left(Y_{i L}+Y_{i U} I_{U}\right)\left(\bar{Y}_{L}+\bar{Y}_{U} I_{L}\right),\left(Y_{i L}+Y_{i U} I_{U}\right)\left(\bar{Y}_{L}+\bar{Y}_{U} I_{U}\right)
\end{array}\right)
\end{array}\right], \quad I_{Y N} \in\left[I_{Y L}, I_{Y U}\right] .
\end{aligned}
$$

Under $H_{0}: \sigma_{1 N}^{2}=\sigma_{2 N}^{2}$, the neutrosophic $F_{N} \in\left[F_{L}, F_{U}\right]$ is defined by

$$
F_{N}=\frac{S_{Y N}^{2}}{S_{X N}^{2}} ; \quad F_{N} \in\left[F_{L}, F_{U}\right] .
$$

The neutrosophic correlation defined by the work in [34] is given by

$$
r_{N}=\frac{n_{N} \sum X_{n N} Y_{n N}-\sum X_{n N} \sum Y_{n N}}{\sqrt{\left\{n_{N} \sum X_{n N}^{2}-\left(\sum X_{n N}\right)^{2}\right\}\left\{n_{N} \sum X_{n N}^{2}-\left(\sum Y_{n N}^{2}\right)^{2}\right\}}} ; \quad n_{N} \in\left[n_{L}, n_{U}\right] .
$$


The neutrosophic quotient $\gamma_{N} F_{N} \in\left[\gamma_{L} F_{U}, \gamma_{L} F_{U}\right]$ is defined by

$$
\gamma_{N} F_{N}=\frac{F_{N}-1}{\left[\left(F_{N}+1\right)^{2}-4 r_{N}^{2} F_{N}\right]} ; \quad \gamma_{N} F_{N} \in\left[\gamma_{L} F_{U}, \gamma_{L} F_{U}\right] .
$$

The neutrosophic form of $\gamma_{N} F_{N} \in\left[\gamma_{L} F_{U}, \gamma_{L} F_{U}\right]$ can be expressed as

$$
\gamma_{N} F_{N}=\gamma_{L} F_{L}+\gamma_{U} F_{U} I_{\gamma_{N} F_{N}} ; \quad I_{\gamma_{N} F_{N}} \in\left[I_{\gamma_{L} F_{L}}, I_{\gamma_{U} F_{U}}\right] .
$$

In the neutrosophic form of $\gamma_{N} F_{N} \in\left[\gamma_{L} F_{U}, \gamma_{L} F_{U}\right]$, the first part $\gamma_{L} F_{L}$ presents the determined part and $\gamma_{U} F_{U} I_{\gamma_{N} F_{N}}$ is the indeterminate part. The proposed form of quotient reduces to classical statistics when $I_{\gamma_{L} F_{L}}=0$. The application of the proposed test is discussed using Figure 1.

\section{Application Using Food Data}

The application of the proposed test will be given on the data collected from the food industry. To keep the quality of the food, the food inspectors test the food for different characteristics such as taste, shape, and hardness. Similar examples were discussed in $[22,38]$. The evaluation of food by the inspector for product $A$ and product $B$ is shown in Table 1. From Table 1, it is clear that experts provide the food evaluation in indeterminate interval reporting the minimum value and the maximum value. The evaluation of food is imprecise data rather than the exact; therefore, the existing test is under classical statistics. For the data, the decision makers are interested to see whether the variances of both products have the same variances or not. Therefore, the proposed test can be applied to test $H_{0}: \sigma_{1 N}^{2}=\sigma_{2 N}^{2}$ vs. the alternative hypothesis $H_{1}: \sigma_{1 N}^{2} \neq \sigma_{2 N}^{2}$. The neutrosophic means for the data are given by

$$
\begin{aligned}
& \bar{X}_{i N}=5.84+6.86 I_{X N} ; \quad I_{X N} \in[0,0.1486], \\
& \bar{Y}_{i N}=8.26+9.0 I_{Y N} ; \quad I_{Y N} \in[0,0.0822] .
\end{aligned}
$$

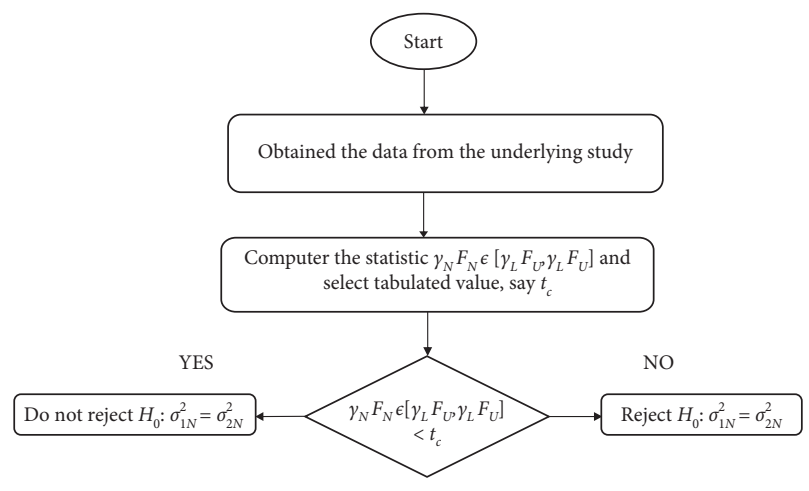

Figure 1: The operational process of the proposed test.

The neutrosophic variances for the data are calculated as follows:

$$
\begin{aligned}
& S_{X N}^{2}=\frac{\sum_{i=1}^{n_{N}}\left(X_{i N}-\bar{X}_{i N}\right)^{2}}{n_{N}}=\frac{[4.6,11]}{10}=[0.46,1.1], \\
& S_{Y N}^{2}=\frac{\sum_{i=1}^{n_{N}}\left(Y_{i N}-\bar{Y}_{i N}\right)^{2}}{n_{N}}=\frac{[4.904,8.28]}{10}=[0.49,0.828] .
\end{aligned}
$$

Under $H_{0}: \sigma_{1 N}^{2}=\sigma_{2 N}^{2}$, the neutrosophic $F_{N} \in\left[F_{L}, F_{U}\right]$ is calculated as

$$
F_{N}=\frac{S_{Y N}^{2}}{S_{X N}^{2}}=\frac{[0.49,0.828]}{[0.46,1.1]} ; \quad F_{N} \in[1.135,1.765] .
$$

The neutrosophic correlation is calculated as $r_{N} \in[0.02,-0.1785]$. The neutrosophic quotient $\gamma_{N} F_{N} \in\left[\gamma_{L} F_{U}, \gamma_{L} F_{U}\right]$ is calculated as

$$
\gamma_{N} F_{N}=\frac{[1.135,1.765]-1}{\left[([1.135,1.765]+1)^{2}-4[0.02,-0.1785][1.135,1.765]\right]} ; \quad \gamma_{N} F_{N} \in[0.06,0.28]
$$

The neutrosophic form of $\gamma_{N} F_{N} \in\left[\gamma_{L} F_{U}, \gamma_{L} F_{U}\right]$ is given as

$$
\gamma_{N} F_{N}=0.06+0.28 I_{\gamma_{N} F_{N}} ; \quad I_{\gamma_{N} F_{N}} \in[0,0.7857] .
$$

In the neutrosophic form of $\gamma_{N} F_{N} \in\left[\gamma_{L} F_{U}, \gamma_{L} F_{U}\right]$, the first part 0.06 presents the determined part and $0.28 I_{\gamma_{N} F_{N}}$ is the indeterminate part, where $I_{\gamma_{N} F_{N}} \in[0,0.7857]$ is the measure of indeterminacy associated with $\gamma_{N} F_{N} \in\left[\gamma_{L} F_{U}, \gamma_{L} F_{U}\right]$. The proposed form of quotient reduces to classical statistics when
$I_{\gamma_{L} F_{L}}=0$. The proposed test is implemented in the following steps:

Step- 1: state $H_{0}: \sigma_{1 N}^{2}=\sigma_{2 N}^{2}$ vs. $H_{1}: \sigma_{1 N}^{2} \neq \sigma_{2 N}^{2}$

Step- 2: set the level of significance $\alpha=0.05$

Step- 3: calculate $\gamma_{N} F_{N} \in[0.06,0.28]$, and compared with the critical value from [1], it is 0.632

Step- 4: as $\gamma_{N} F_{N} \in[0.06,0.28]<0.632$, the null hypothesis $H_{0}: \sigma_{1 N}^{2}=\sigma_{2 N}^{2}$ can be accepted 
TABLE 1: The neutrosophic data.

\begin{tabular}{|c|c|c|c|c|c|c|c|c|c|c|}
\hline$A=X_{i N}$ & {$[5,7]$} & {$[6.5,7.5]$} & {$[5,7]$} & {$[6,8]$} & {$[7,9.4]$} & {$[6.5,8.1]$} & {$[5,8.4]$} & {$[6,9]$} & {$[5.4,7.8]$} & {$[6,8.4]$} \\
\hline$B=Y_{i N}$ & {$[7,9]$} & {$[8.2,10]$} & {$[6.5,8.3]$} & {$[6,8]$} & {$[5.5,8.3]$} & {$[7,9]$} & {$[6.4,10]$} & {$[8,10]$} & {$[6.8,8.8]$} & {$[7.2,8.6]$} \\
\hline
\end{tabular}

From the study, it is concluded that the neutrosophic variances of both food experts are the same. Therefore, there is no significant difference between the variances.

\section{Comparative Study Based on Food Data}

It is noted that the proposed test is a generalization of the existing test under neutrosophic statistics. It is also worth noting that the proposed test reduces to the existing test under classical statistics when no indeterminacy is found in the data. Therefore, the efficiency of the proposed test will be given in terms of the measure of indeterminacy, information, and flexibility. The neutrosophic form of $\gamma_{N} F_{N} \in\left[\gamma_{L} F_{U}, \gamma_{L} F_{U}\right]$ is given as $\gamma_{N} F_{N}=0.06+0.28 I_{\gamma_{N} F_{N}} ; I_{\gamma_{N} F_{N}} \in[0,0.7857]$. As discussed earlier, the first part 0.06 shows the value of statistic under classical statistics, the second part $0.28 I_{\gamma_{N} F_{N}}$ presents the indeterminate part, and $I_{\gamma_{N} F_{N}} \in[0,0.7857]$ is the measure of uncertainty associated with the statistic $\gamma_{N} F_{N} \in\left[\gamma_{L} F_{U}, \gamma_{L} F_{U}\right]$. The proposed test statistic becomes the existing test statistic when $\gamma_{L} F_{U}=0$. From the study, it can be noted that the proposed test statistic has $\gamma_{N} F_{N} \in[0.06,0.28]$. It means, under an uncertain environment, the decision makers can expect the value of statistics from 0.06 to 0.28 . On the other hand, the existing statistic gives only the determined value which is not adequate under an indeterminate environment. Therefore, the proposed test is more flexible than the existing test. In addition to this flexibility, the proposed test provides additional information about the measure of indeterminacy that is 0.7857 . The proposed test gives information about three events associated with $H_{0}: \sigma_{1 N}^{2}=\sigma_{2 N}^{2}$. The probability of accepting $H_{0}: \sigma_{1 N}^{2}=\sigma_{2 N}^{2}$ is 0.95 , the probability of committing of a type-I error (the probability of rejecting $H_{0}: \sigma_{1 N}^{2}=\sigma_{2 N}^{2}$ when it is true) is 0.05 , and the chance of indeterminacy is 0.7857 . From this comparison, it is clear that the proposed test gives more information, suitable, and flexible than the existing test. Therefore, the decision makers can apply the proposed test for testing $H_{0}: \sigma_{1 N}^{2}=\sigma_{2 N}^{2}$ under indeterminacy.

\section{Concluding Remarks}

The present study introduced the neutrosophic statistical test to investigate the difference between variances of two populations when the correlation was exsiting in pair observations. The procedure and statistic of the proposed test under neutrosophic statistics were introduced in this paper. The proposed test is an extension of the existing F-test when correlation exists in pair observations. The application of the proposed test was given using the food industry data. The comparative study showed the efficiency of the proposed test over the existing test in terms of knowledge, flexibility, and adequacy. The proposed test using big data can be considered as future research. The proposed test using various sampling schemes can also be considered for future research.

\section{Data Availability}

The data used to support the findings of this study are included within the article.

\section{Conflicts of Interest}

The authors declare no conflicts of interest.

\section{Acknowledgments}

This work was supported by the Deanship of Scientific Research (DSR), King Abdulaziz University, Jeddah, under Grant no. GP: 142-130-1442. The authors, therefore, gratefully acknowledge the DSR technical and financial support.

\section{References}

[1] G. K. Kanji, 100 Statistical Tests, SAGE, Thousand Oaks, CA, USA, 2006.

[2] F. Ceriotti, D. Brugnoni, and S. Mattioli, "How to define a significant deviation from the expected internal quality control result," Clinical Chemistry and Laboratory Medicine, vol. 53, no. 6, pp. 913-918, 2015.

[3] T. Hashimoto, "Sample size determination given data of preliminary experiment for Student's t-test, ANOVA and Tukey's multiple comparison," Japanese Journal of Psychology, vol. 75, no. 3, pp. 213-219, 2004.

[4] J. O. Westgard and M. R. Hunt, "Use and interpretation of common statistical tests in method-comparison studies," Clinical Chemistry, vol. 19, no. 1, pp. 49-57, 1973.

[5] M. Flores, R. Fernández-Casal, S. Naya, J. Tarrío-Saavedra, and R. Bossano, "ILS: an R package for statistical analysis in Interlaboratory Studies," Chemometrics and Intelligent Laboratory Systems, vol. 181, pp. 11-20, 2018.

[6] S. Maghsoodloo and C.-Y. Huang, "Comparing the overlapping of two independent confidence intervals with a single confidence interval for two normal population parameters," Journal of Statistical Planning and Inference, vol. 140, no. 11, pp. 3295-3305, 2010.

[7] S. Niwitpong and S.-a. Niwitpong, "Confidence interval for the difference of two normal population means with a known ratio of variances," Applied Mathematical Sciences, vol. 4, no. 8, pp. 347-359, 2010.

[8] M. Regis, T. A. Postma, and E. R. van den Heuvel, "A note on the calculation of reference change values for two consecutive normally distributed laboratory results," Chemometrics and Intelligent Laboratory Systems, vol. 171, pp. 102-111, 2017.

[9] R. Viertl, "Univariate statistical analysis with fuzzy data," Computational Statistics \& Data Analysis, vol. 51, no. 1, pp. 133-147, 2006. 
[10] J. Chachi, S. M. Taheri, and R. Viertl, "Testing statistical hypotheses based on fuzzy confidence intervals," Austrian Journal of Statistics, vol. 41, no. 4, pp. 267-286, 2012.

[11] W. S. Du, "Correlation and correlation coefficient of generalized orthopair fuzzy sets," International Journal of Intelligent Systems, vol. 34, no. 4, pp. 564-583, 2019.

[12] P. Filzmoser and R. Viertl, "Testing hypotheses with fuzzy data: the fuzzy $p$ value," Metrika, vol. 59, no. 1, pp. 21-29, 2004.

[13] H. Garg and D. Rani, "New generalised Bonferroni mean aggregation operators of complex intuitionistic fuzzy information based on Archimedean t-norm and t-conorm," Journal of Experimental \& Theoretical Artificial Intelligence, vol. 32, no. 1, pp. 81-109, 2020.

[14] P. Grzegorzewski, "k-sample median test for vague data," International Journal of Intelligent Systems, vol. 24, no. 5, pp. 529-539, 2009.

[15] P. Grzegorzewski and M. Śpiewak, "The sign test and the signed-rank test for interval-valued data," International Journal of Intelligent Systems, vol. 34, no. 9, pp. 2122-2150, 2019.

[16] E. B. Jamkhaneh and A. N. Ghara, "Testing statistical hypotheses with fuzzy data," in Proceedings of the International Conference on Intelligent Computing and Cognitive Informatics, Washington, DC, USA, June 2010.

[17] D. Kalpanapriya and P. Pandian, "Statistical hypotheses testing with imprecise data," Applied Mathematical Sciences, vol. 6, no. 106, pp. 5285-5292, 2012.

[18] M. Montenegro, M. a. R. Casals, M. a. A. Lubiano, and M. a. A. Gil, "Two-sample hypothesis tests of means of a fuzzy random variable," Information Sciences, vol. 133, no. 1-2, pp. 89-100, 2001.

[19] S. Park, S.-J. Lee, and S. Jun, "Patent big data analysis using fuzzy learning," International Journal of Fuzzy Systems, vol. 19, no. 4, pp. 1158-1167, 2017.

[20] S. Singh and A. H. Ganie, "On some correlation coefficients in Pythagorean fuzzy environment with applications," International Journal of Intelligent Systems, vol. 35, no. 4, pp. 682-717, 2020.

[21] S. M. Taheri and M. Arefi, “Testing fuzzy hypotheses based on fuzzy test statistic," Soft Computing, vol. 13, no. 6, pp. 617-625, 2009.

[22] C.-C. Tsai and C.-C. Chen, "Tests of quality characteristics of two populations using paired fuzzy sample differences," International Journal of Advanced Manufacturing Technology, vol. 27, no. 5, pp. 574-579, 2006.

[23] F. Smarandache, Neutrosophy. Neutrosophic Probability, Set, and Logic, vol. 105, pp. 118-123, ProQuest Information \& Learning, Ann Arbor, MI, USA, 1998.

[24] F. Smarandache, Introduction To Neutrosophic Measure, Neutrosophic Integral, and Neutrosophic Probability, Infinite Study, Darya Ganj, India, 2013.

[25] M. Abdel-Baset, V. Chang, and A. Gamal, "Evaluation of the green supply chain management practices: a novel neutrosophic approach," Computers in Industry, vol. 108, pp. 210-220, 2019.

[26] M. Abdel-Basset, M. Mohamed, M. Elhoseny, L. H. Son, and F. Chiclana, "Cosine similarity measures of bipolar neutrosophic set for diagnosis of bipolar disorder diseases," Artificial Intelligence in Medicine, vol. 101, Article ID 101735, 2019.

[27] S. Broumi, A. Bakali, M. Talea, and F. Smarandache, Bipolar Neutrosophic Minimum Spanning Tree, Infinite Study, Darya Ganj, India, 2018.
[28] S. Broumi and F. Smarandache, "Correlation coefficient of interval neutrosophic set," Applied Mechanics and Materials, vol. 436, 2013.

[29] Y. Guo and A. Sengur, "NCM: neutrosophic c-means clustering algorithm," Pattern Recognition, vol. 48, no. 8, pp. 2710-2724, 2015.

[30] F. Smarandache, Introduction To Neutrosophic Statistics, Infinite Study, Darya Ganj, India, 2014.

[31] M. Zaied, "Neutrosophic analysis of variance: application to university students," Complex \& Intelligent Systems, vol. 5, 2019.

[32] M. Aslam, "A new attribute sampling plan using neutrosophic statistical interval method," Complex \& Intelligent Systems, vol. 5, 2019.

[33] M. Aslam, "A new method to analyze rock joint roughness coefficient based on neutrosophic statistics," Measurement, vol. 146, pp. 65-71, 2019.

[34] M. Aslam and M. Albassam, "Application of neutrosophic logic to evaluate correlation between prostate cancer mortality and dietary fat assumption," Symmetry, vol. 11, no. 3, p. 330, 2019.

[35] J. Chen, J. Ye, and S. Du, "Scale effect and anisotropy analyzed for neutrosophic numbers of rock joint roughness coefficient based on neutrosophic statistics," Symmetry, vol. 9, no. 10, p. 208, 2017.

[36] J. Chen, J. Ye, S. Du, and R. Yong, "Expressions of rock joint roughness coefficient using neutrosophic interval statistical numbers," Symmetry, vol. 9, no. 7, p. 123, 2017.

[37] J. Pratihar, R. Kumar, S. Edalatpanah, and A. Dey, "Modified Vogel's approximation method for transportation problem under uncertain environment," Complex \& Intelligent Systems, vol. 7, pp. 29-40, 2020.

[38] P. Grzegorzewski, "Two-sample dispersion problem for fuzzy data," in Proceedings of the International Conference on Information Processing and Management of Uncertainty in Knowledge-Based Systems, Lisbon, Portugal, June 2020. 